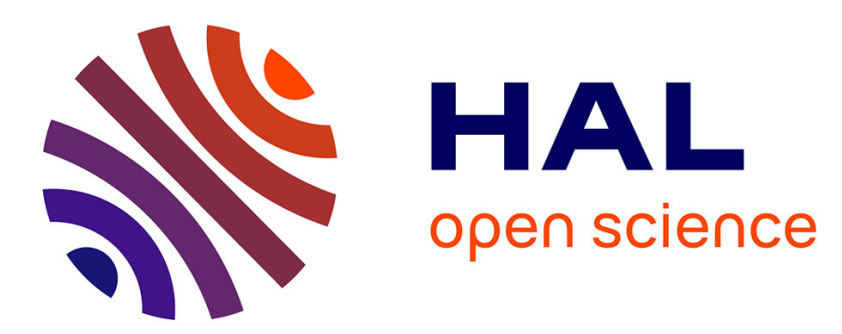

\title{
Amplitude Independent Frequency Synchroniser for a Cubic Planar Polynomial System
}

\author{
Islam Boussaada
}

\section{To cite this version:}

Islam Boussaada. Amplitude Independent Frequency Synchroniser for a Cubic Planar Polynomial System. Journal of Computational and Nonlinear Dynamics, 2012, http://computationalnonlinear.asmedigitalcollection.asme.org/article.aspx?articleid=1397028. 10.1115/1.4005322 . hal-01123578

\section{HAL Id: hal-01123578 https://hal.science/hal-01123578}

Submitted on 5 Mar 2015

HAL is a multi-disciplinary open access archive for the deposit and dissemination of scientific research documents, whether they are published or not. The documents may come from teaching and research institutions in France or abroad, or from public or private research centers.
L'archive ouverte pluridisciplinaire HAL, est destinée au dépôt et à la diffusion de documents scientifiques de niveau recherche, publiés ou non, émanant des établissements d'enseignement et de recherche français ou étrangers, des laboratoires publics ou privés. 


\title{
Amplitude Independent Frequency Synchroniser for a Cubic Planar Polynomial System
}

\author{
Islam Boussaada
}

\begin{abstract}
The problem of local linearizability of the planar linear center perturbed by cubic nonlinearities in all generalities on the system parameters (14 parameters) is far from being solved. Synchronization problem $[18,5]$ consists in bringing appropriate modifications on a given system to obtain a desired dynamic. The desired phase portrait along this paper contains a compact region around a singular point at the origin in which lies periodic orbits with the same period (independently from the chosen initial conditions). In this paper, starting from a 5-parameters non isochronous Chouikha cubic system [8], we identify all possible monomial perturbations of degree $d \in\{2,3\}$ insuring local linearizability of the perturbed system. The necessary conditions are obtained by the Normal Forms method. These conditions are real algebraic equations (multivariate polynomials) in the parameters of the studied ordinary differential system. The efficient algorithm FGb [13] for computing the Gröbner basis is used. For the family studied in this paper, an exhaustive list of possible parameters values insuring local linearizability is established. All the found cases are already known in the literature but the contexts is different since our object is the synchronisation not the classification. This paper can be seen as a direct continuation of several new works concerned with the hinting of cubic isochronous centers in particular $[8,12,15,10,6,2,3]$, also it can be considered as an adaptation of a qualitative theory method to a synchronization problem.
\end{abstract}

Mathematics Subject Classification (2010). Primary 34C15, 34C25, 34C37.

Keywords. Frequency synchronization, Amplitude independent, Center conditions, Period function, Normal forms, Isochronicity, Linearizability, Polynomial systems, Symbolic computations, Gröbner basis, FGb algorithm, Maple.

\section{Introduction}

We consider the planar dynamical system,

$$
\frac{d x}{d t}=\dot{x}=X(x, y), \quad \frac{d y}{d t}=\dot{y}=Y(x, y),
$$

where $(x, y)$ belongs to an open connected subset $U \subset \mathbb{R}^{2}, X, Y \in C^{k}(U, \mathbb{R})$, and $k \geq 1$. Due to Poincaré : an isolated singular point $p \in U$ of (1.1) is a center if and only if there exists a punctured neighborhood $V \subset U$ of $p$ such that every orbit in $V$ is a cycle surrounding $p$. A center is said to be isochronous if all the orbits surrounding it have the same period. An overview of J.Chavarriga and

Journal of Computational and Nonlinear Dynamics, (ASME), April 2012, Volume 7, Issue 2, 021009, (8 pp). 
M.Sabatini [7] present the methods and basic results concerning the problem of the isochronicity, see also $[14,8,3,6,2]$.

Synchronization problem consists in bringing appropriate modifications on a given system to obtain a desired dynamic, see $[18,5]$. Along this paper, the desired phase portrait contains a compact region around a singular point at the origin in which lies periodic orbits with the same period (independently from the chosen initial conditions which is not always the case). More concretely, in this paper we consider the following problem; Starting from a non isochronous polynomial planar system, are there any polynomial perturbation which insures the local linearisability of the perturbed system. In this paper we adopt the normal forms method often used in qualitative theory investigations; center-focus problem, bifurcation problem and local linearisability problem. The problem of local linearisability conditions of of the planar linear center perturbed by cubic nonlinearities (in all generalities on the system parameters 14 parameters) is far to be solved.

In this paper, starting from a 5-parameters non isochronous Chouikha cubic system [8], we identify all possible monomial perturbations of degree $d \in\{2,3\}$ insuring local linearizability of the perturbed system. Investigations are based on the normal forms Theory.

In the following system as well as in all other considered systems, all parameters are reals.

Consider the real Liènard Type equation

$$
\ddot{x}+f(x) \dot{x}^{2}+g(x)=0
$$

or equivalently its associated two dimensional (planar) system

$$
\left.\begin{array}{l}
\dot{x}=y \\
\dot{y}=-g(x)-f(x) y^{2}
\end{array}\right\}
$$

The study of isochronicity of (1.2) was established first in M. Sabatini paper [21]. The sufficient conditions of the isochronicity of the origin $O$ for system (1.3) with $f$ and $g$ of class $C^{1}$ are given. In the analytic case, the necessary and sufficient conditions for isochronicity are given by A.R.Chouikha in [8]. In the same paper, the author implemented a new algorithmic method for computing isochronicity conditions for system (1.3) called C-algorithm. As an application of this algorithm, the author studied the following cubic system

$$
\left.\begin{array}{l}
\dot{x}=-y+\tilde{a}_{1,2,1} x^{2} y \\
\dot{y}=x+\tilde{a}_{2,2,0} x^{2}+\tilde{a}_{2,0,2} y^{2}+\tilde{a}_{2,3,0} x^{3}+\tilde{a}_{2,1,2} x y^{2}
\end{array}\right\}
$$

where all the parameters values for which system (1.4) has an isochronous center at the origin are established in the following theorem.

We note that the coefficient $a_{i, j, k}$ denotes the parameter of the monomial perturbation of the $i^{t h}$ equation of the linear isochronous center $(\dot{x}=-y, \dot{y}=x)$ of degree $j$ in $x$ and of degree $k$ in $y$

Theorem 1.1 (Chouikha,[8]). System (1.4) has an isochronous center at 0 if and only if its parameters satisfy one of the following conditions

1. $\tilde{a}_{2,3,0}=-(2 / 3) \tilde{a}_{1,2,1}, \tilde{a}_{2,1,2}=3 \tilde{a}_{1,2,1}, \tilde{a}_{2,2,0}=\tilde{a}_{2,0,2}=0$

2. $\tilde{a}_{2,1,2}=\tilde{a}_{1,2,1}, \tilde{a}_{2,2,0}=\tilde{a}_{2,3,0}=\tilde{a}_{2,0,2}=0$

3. $\tilde{a}_{2,3,0}=(1 / 14) \tilde{a}_{2,0,2}^{2}, \tilde{a}_{2,1,2}=(3 / 7) \tilde{a}_{2,0,2}^{2}, \tilde{a}_{1,2,1}=(1 / 7) \tilde{a}_{2,0,2}^{2}, \tilde{a}_{2,2,0}=-(1 / 2) \tilde{a}_{2,0,2}$

4. $\tilde{a}_{2,1,2}=\tilde{a}_{2,0,2}^{2}, \tilde{a}_{2,3,0}=0, \tilde{a}_{1,2,1}=(1 / 2) \tilde{a}_{2,0,2}^{2}, \tilde{a}_{2,2,0}=-(1 / 2) \tilde{a}_{2,0,2}$.

A 1-parameter perturbation of system (1.4) is studied in [10]. Namely, the following system

$$
\left.\begin{array}{l}
\dot{x}=-y+\underline{a_{1,1,1} x y}+a_{1,2,1} x^{2} y \\
\dot{y}=x+a_{2,2,0} x^{2}+a_{2,0,2} y^{2}+a_{2,3,0} x^{3}+a_{2,1,2} x y^{2}
\end{array}\right\}
$$

which is system (1.4) perturbed by the underlined term. All the values of the parameters for which the above system (1.5) has an isochronous center at the origin were found. 
Note that the above system stills reducible to the Liénard type equation for wich C-algorithm is applicable, see [6].

Section 2 is devoted to recall the head lines of the methodology of the Normal Forms algorithm, called in the sequel NF algorithm, which will allow us to obtain isochronicity necessary conditions.

The last section is concerned with the main result, which is an application of the NF method. Indeed, we consider an unknown 1-directional monomial perturbation of degree two or three of the system (1.4), namely

$$
\left.\begin{array}{l}
\dot{x}=-y+a_{1,2,1} x^{2} y+\underline{\Psi_{1}(x, y)} \\
\dot{y}=x+a_{2,2,0} x^{2}+a_{2,0,2} y^{2}+a_{2,3,0} x^{3}+a_{2,1,2} x y^{2}+\underline{\Psi_{2}(x, y)}
\end{array}\right\},
$$

in which only one of the monomials $\Psi_{1}$ or $\Psi_{2}$ is non zero monomial $\left(\Psi_{1} \Psi_{2}=0\right)$ of degree $d \in$ $\{2,3\}$.

The problem turns to study eight polynomial cubic systems which are not reducible by the transformations described in [6] to Liénard type equation. For each system, we identify the values of the parameters for which the singular point at thea origin is an isochronous center. Hence it is done for (1.6).

\section{The normal forms method}

The normal form theory which is due essentially to Poincaré, presents a basic tool in understanding the qualitative behavior of orbit structures of a vector field near equilibria [16]. It was used for the study of center conditions and the nature of bifurcation of a given vector field. We also recall a pioneer work in this field established by Pleshkan (see[19]), in which the author presented an investigation method of isochronicity in the case of linear center perturbed by homogeneous cubic nonlinearity. The principle of Pleshkan's algorithm is very close to the one presented in Algaba et.al paper [1], where the normal form theory is used in the analysis of isochronicity and gave a recursive method for the isochronicity investigation. In the last cited paper, the autors studied cubic Liénard equation and obtained a characterization of the reversible Liénard equation having an isochronous center at the origin.

Let $x=\left(x_{1}, x_{2}\right) \in \mathbb{R}^{2}$ and $f\left(x_{1}, x_{2}\right) \in \mathbb{R}\left[x_{1}, x_{2}\right] \times \mathbb{R}\left[x_{1}, x_{2}\right]$ and consider the general planar system

$$
\dot{x}=L x+f(x)=L x+f_{2}(x)+f_{3}(x)+\ldots+f_{k}(x)+\ldots,
$$

where $L x$ represents the linear part, $L$ the Jacobian matrix associated to system $(2.1)$ and $f_{k}(x)$ denotes the $k^{t h}$ order vector homogeneous polynomials of $x$. We assume that the system admits an equilibrium at the origin $O$. The essential idea of the Normal Form theory is to find a near identity transformation

$$
x=y+h(y)=y+h_{2}(y)+h_{3}(y)+\ldots+h_{k}(y)+\ldots,
$$

by which the resulting system

$$
\dot{y}=L y+g(x)=L y+g_{2}(y)+g_{3}(y)+\ldots+g_{k}(y)+\ldots,
$$

becomes as simple as possible. In this sense, the terms that are not essential in the local dynamical behavior are removed from the analytical expression of the vector field. Let us denote by $h_{k}(y)$ and $g_{k}(y)$ the $k^{t h}$ order vectors homogeneous polynomials of $y$. According to Takens normal form theory, we define an operator as follows:

$$
L_{k}: H_{k} \rightarrow H_{k}, \quad U_{k} \in H_{k} \mapsto L_{k}\left(U_{k}\right)=\left[U_{k}, u_{1}\right] \in H_{k}
$$


where $u_{1}=L y$ is the linear part of the vector field and $H_{k}$ denotes a linear vector space containing the $k^{t h}$ degree homogeneous vector polynomials of $y=\left(y_{1}, y_{2}\right)$. The operator [.,.] is called the Lie Bracket, defined by

$$
\left[U_{k}, u_{1}\right]=L U_{k}-D\left(U_{k}\right) u_{1}
$$

where $D$ denotes the frechet derivative.

Next, we define the spaces $R_{k}$ and $K_{k}$ as the range of $L_{k}$ and the complementary space of $R_{k}$ respectively. Thus, $H_{k}=R_{k}+K_{k}$ and one can then choose bases for $K_{k}$ and $R_{k}$. The normal form theorem determines how it is possible to reduce the analytic expression of the vector field (see Gukenheimer-Holmes [16]). The authors give explicitly an analysis for the quadratic and the cubic cases. Consequently, a vector homogeneous polynomial $f_{k} \in H_{k}$ can be split into two parts, such that one of them can be spanned in $K_{k}$ and the remaining part in $R_{k}$.

Normal form theory shows that the part belonging to $R_{k}$ can be eliminated and the remaining part can be retained in the normal form. By the equations (2.1), (2.2) and (2.3), we can obtain algebraic equations one order after another.

Theorem 2.1 (Yu et. al.,[24]). The recursive formula for computing the normal form coefficients and the nonlinear transformation are given by:

$$
\begin{gathered}
g_{k}=f_{k}+\left[h_{k}, L y\right]+\sum_{i=2}^{k-1}\left(D f_{i} h_{k-i+1}-D h_{k-i+1} g_{i}\right) \\
+\sum_{i=2}^{[k / 2]} \frac{1}{i !} \sum_{j=i}^{k-i} D^{i} f_{j} \sum_{l_{1}+l_{2}+\ldots+l_{i}=k-(j-i) 2 \leq l_{1}, l_{2}, \ldots, l_{i} \leq k+2-(i+j)} h_{l_{1}} h_{l_{2}} \ldots h_{l_{i}},
\end{gathered}
$$

for $k=2,3, \ldots$

see also $[22,23]$.

System (2.3) can be transformed to the polar coordinate system with $y_{1}=r \cos (\theta), y_{2}=$ $r \sin (\theta)$ so that

$$
\dot{r}=\sum_{j=1}^{N} \alpha_{2 j+1} r^{2 j+1}+O\left(r^{2 N+3}\right), \dot{\theta}=1+\sum_{j=1}^{N} \beta_{2 j+1} r^{2 j}+O(r 2 N+2) .
$$

Recall that a necessary condition to have a center at the origin is that all the focal values $\alpha_{2 j+1}$ vanish. By the Hilbert's basis theorem, the set of focal values has a finite basis in the ring of polynomials in the coefficients of the initial system (2.1). Since the non vanishing of one of the angular component coefficient implies dependency of an associated period constant, a necessary condition for which this center is isochronous is that $\beta_{2 j+1}$ vanish.

Recall that our study is motivated by the interest of describing a synchronizer for a desired dynamic but also to underline the key role that can the classification of centers and isochronous centers of polynomial systems have in applications such that synchronization problems.

\section{Main results :}

\section{Applications of the NF algorithm to cubic systems}

In our study, we use Maple. To compute the Gröbner basis of the obtained polynomial equations in the ring of characteristic 0, we employ the Salsa Software [13]. More precisely we use the $F G b$ algorithm which is the most efficient algorithm in computing Gröbner basis [4], at least for the polynomial systems studied in this paper. We note also that we have used DRL ordering in all computations established in this paper. 
Since our approach in investigation of isochronicity conditions is based on an algorithmic method, then we can guess that every simplification is beneficial in the goal of speeding the computations and reducing the necessary memory size. Solving multivariate algebraic equations (real polynomials) can be a very hard task if we try to manipulate the polynomial equations without tricks. Interested readers can find in the website of Salsa Software [13], more precisely the page of J.C.Faugère, some important rules in solving polynomial systems and about Gröbner basis.

Let us consider the more general cubic perturbation of linear center :

$$
\left.\begin{array}{l}
\dot{x}=y+a_{1,2,0} x^{2}+a_{1,0,2} y^{2}+a_{1,1,1} x y+a_{1,3,0} x^{3}+a_{1,2,1} x^{2} y+a_{1,1,2} x y^{2}+a_{1,0,3} y^{3} \\
\dot{y}=-x+a_{2,2,0} x^{2}+a_{2,0,2} y^{2}+a_{2,1,1} x y+a_{2,30} x^{3}+a_{2,2,1} x^{2} y+a_{2,1,2} x y^{2}+a_{2,0,3} y^{3}
\end{array}\right\}
$$

or equivalently the following one :

$$
\left.\begin{array}{l}
\dot{x}=-y+\tilde{a}_{1,2,0} x^{2}+\tilde{a}_{1,0,2} y^{2}+\tilde{a}_{1,1,1} x y+\tilde{a}_{1,3,0} x^{3}+\tilde{a}_{1,2,1} x^{2} y+\tilde{a}_{1,1,2} x y^{2}+\tilde{a}_{1,0,3} y^{3} \\
\dot{y}=x+\tilde{a}_{2,2,0} x^{2}+\tilde{a}_{2,0,2} y^{2}+\tilde{a}_{2,1,1} x y+\tilde{a}_{2,30} x^{3}+\tilde{a}_{2,2,1} x^{2} y+\tilde{a}_{2,1,2} x y^{2}+\tilde{a}_{2,0,3} y^{3}
\end{array}\right\} .
$$

Observe that we can easily reconstruct the coefficient $\tilde{a}_{i, j, k}$ from the ones in (3.1) by the change of coordinates $(x, y) \mapsto(-x, y)$.

The classification of all the isochronous centers of the above system is a very hard task. By any recursive method from those quoted in [7], solving the isochronicity problem for system (3.1) is very difficult in the sense of solving multivariate polynomials. Here, the variables are the 14 parameters of the polynomial differential system (3.1). Hence it needs very important computation supports.

With a realistic point of view, several authors have chosen some particular cases of the above system for investigation like the homogeneous cubic perturbations of the linear center $[19,7]$ and time reversible cubic systems $[12,15]$.

In our case, we focus on an unknown 1-directional 1-parameter perturbation of the system (1.4) which is system (1.6). Therefore, we ramify our study to all possible cases.

In the sequel, every subsection will be concerned with a possible 1-parameter perturbation of system (1.4).

Recall that $a_{i, j, k}$ denotes the parameter of the monomial perturbation of the $i^{t h}$ equation in system (1.4) of degree $j$ in $x$ and of degree $k$ in $y$.

\section{1. perturbation $a_{1,1,1}$}

As a continuation of the result of [8] on system (1.4), Chouikha, Romanovski and Chen investigated a 1-parameter perturbation of (1.4) which is (1.5).

Theorem 3.1 (Chouikha et al.[10]). System (1.5) has an isochronous center at the origin $O$ if and only if

$$
a_{1,2,1}=\frac{-a_{1,1,1}+a_{1,1,1} a_{2,2,0}-10 a_{2,2,0}^{2}+5 a_{1,1,1} a_{2,0,2}-10 a_{2,0,2} a_{2,0,2}-4 a_{2,0,2}^{2}+9 a_{2,3,0}+3 a_{2,1,2}}{3} \text { and it's }
$$

parameters satisfy one of the following sets of conditions

1. $2 a_{2,2,0}+a_{2,0,2}-a_{1,1,1}=0$

$a_{2,0,2}^{2}-3 a_{2,0,2} a_{1,1,1}+2 a_{1,1,1}^{2}-6 a_{2,3,0}-\frac{4 a_{2,1,2}}{3}=0$

$a_{2,0,2} a_{2,3,0}-3 a_{2,3,0} a_{1,1,1}+\frac{a_{2,1,2} a_{1,1,1}}{6}-\frac{a_{2,0,2} a_{2,1,2}}{6}=0$

$a_{2,3,0}^{2} a_{1,1,1}^{2}-3 a_{2,3,0}^{2}+\frac{a_{1,1,1}^{2} a_{2,3,0} a_{2,1,2}}{6}+\frac{a_{2,3,0}^{2} a_{2,1,2}}{3}+\frac{5 a_{2,1,2}^{2} a_{2,3,0}}{36}-\frac{a_{2,1,2}^{3}}{5}=0$

$a_{2,1,2} a_{1,1,1} a_{2,0,2}+6 a_{2,3,0} a_{1,1,1}^{2}-a_{2,1,2} a_{1,1,1}^{2}-18 a_{2,3,0}^{2}-a_{2,3,0} a_{2,1,2}+\frac{2 a_{2,1,2}^{2}}{3}=0$

2. $a_{2,1,2}=0$ and

(a) $a_{2,3,0}=a_{2,0,2}-1 / 4 a_{1,1,1}=a_{2,2,0}=0$

(b) $4 a_{2,3,0}-3 a_{1,1,1}=a_{2,2,0}+a_{1,1,1}=a_{1,1,1}^{2}-3 a_{2,3,0}=0$

(c) $a_{2,0,2}-2 a_{1,1,1}=a_{2,3,0}-a_{1,1,1}^{2}=a_{2,2,0}+2 a_{1,1,1}=0$

(d) $a_{2,0,2}-1 / 3 a_{1,1,1}=a_{2,2,0}+2 / 3 a_{1,1,1}=9 / 2 a_{2,3,0}-a_{1,1,1}^{2}=0$ 
3. $a_{2,3,0}=0$ and
(a) $a_{2,2,0}+1 / 2 a_{2,0,2}-1 / 2 a_{1,1,1}=a_{2,0,2}^{2}-3 a_{2,0,2} a_{1,1,1}+2 a_{1,1,1}^{2}-a_{2,1,2}=0$
(b) $2 a_{2,0,2}-a_{1,1,1}=2 a_{2,2,0}+a_{1,1,1}=0$
(c) $a_{2,0,2}-a_{1,1,1}=a_{2,2,0}=0$
(d) $a_{2,0,2}=a_{1,1,1}^{2}-9 a_{2,1,2}=a_{2,2,0}=0$

We contribute by classifying the isochronous centers of all the remaining 1-parameter perturbations of system (1.4). Eight systems are studied to do this.

For these perturbations, first, we check if the center (at the origin $O$ ) conditions are satisfied and after we give necessary and sufficient isochronicity conditions depending only on the six real parameters.

\subsection{Perturbation $a_{1,0,3}$}

We are concerned by the following system

$$
\left.\begin{array}{l}
\dot{x}=y+a_{1,2,1} x^{2} y+a_{1,0,3} y^{3} \\
\dot{y}=-x+a_{2,2,0} x^{2}+a_{2,0,2} y^{2}+a_{2,3,0} x^{3}+a_{2,1,2} x y^{2}
\end{array}\right\}
$$

Lemma 3.2. The investigation of isochronicity criteria of system (3.2) reduces to the investigation in the following three cases

1. $a_{1,0,3}=0$

2. $a_{1,0,3}=1$

3. $a_{1,0,3}=-1$

Proof. For the case $a_{1,0,3} \neq 0$, two cases are to be analyzed.

First we assume that $a_{1,0,3}>0$. We use on (3.2) the change of coordinates :

$$
(x, y) \mapsto a_{1,0,3}^{1 / 2}(x, y)
$$

to obtain

$$
\left.\begin{array}{l}
\dot{x}=y+\frac{a_{1,2,1}}{a_{1,0,3}} x^{2} y+y^{3} \\
\dot{y}=-x+\frac{a_{2,2,0}}{a_{1,0,3}^{1 / 2}} x^{2}+\frac{a_{2,0,2}}{a_{1,0,3}^{1 / 2}} y^{2}+\frac{a_{2,3,0}}{a_{1,0,3}} x^{2}+\frac{a_{2,1,2}}{a_{1,0,3}} x y^{2}
\end{array}\right\} .
$$

When the solutions of the isochronicity problem of system (3.4) are established, we can easily reconstruct those of the original system (3.2) by the transformation

$$
(x, y) \mapsto\left(1 / a_{1,0,3}^{1 / 2}\right)(x, y) .
$$

If $a_{1,0,3}<0$, then (3.2) can be written as

$$
\left.\begin{array}{l}
\dot{x}=y+a_{1,2,1} x^{2} y-\tilde{a}_{1,0,3} y^{3} \\
\dot{y}=-x+a_{2,2,0} x^{2}+a_{2,0,2} y^{2}+a_{2,3,0} x^{3}+a_{2,1,2} x y^{2}
\end{array}\right\}
$$

with $-a_{1,0,3}=\tilde{a}_{1,0,3}>0$. Applying the following change of coordinates

$$
(x, y) \mapsto \tilde{a}_{1,0,3}^{1 / 2}(x, y)
$$

yields

$$
\left.\begin{array}{l}
\dot{x}=y+\frac{a_{1,2,1}}{\tilde{a}_{1,0,3}} x^{2} y-y^{3} \\
\dot{y}=-x+\frac{a_{2,2,0}}{\tilde{a}_{1,0,3}^{1 / 2}} x^{2}+\frac{a_{2,0,2}}{\tilde{a}_{1,0,3}^{1 / 2}} y^{2}+\frac{a_{2,3,0}}{\tilde{a}_{1,0,3}} x^{2}+\frac{a_{2,1,2}}{\tilde{a}_{1,0,3}} x y^{2}
\end{array}\right\} .
$$

The reconstruction of the solutions of (3.2) can be obtained from those of (3.7), by the change of coordinates

$$
(x, y) \mapsto\left(1 / \tilde{a}_{1,0,3}^{1 / 2}\right)(x, y) .
$$


Remark 3.3. If one is concerned with quadratic perturbations of system (1.4) with the parameter $a_{i, j, 2-j}$

we can consider the two cases namely,

1. $a_{i, j, 2-j}=0$

2. $a_{i, j, 2-j}=1$

Indeed, when $a_{i, j, 2-j} \neq 0$, the change of coordinates

$$
(x, y) \mapsto a_{i, j, 2-j}(x, y)
$$

reduces the problem to the case $a_{i, j, 2-j}=1$.

Lastly, thanks to the transformation

$$
(x, y) \mapsto 1 / a_{i, j, 2-j}(x, y),
$$

we can easily reconstruct the solutions of the problem when $a_{i, j, 2-j} \neq 0$.

Theorem 3.4. System (3.2) has an isochronous center at $O$ if and only if its parameters satisfy one of the following conditions :

1. $a_{1,0,3}=0$

(a) $a_{1,2,1}=a_{2,1,2}, a_{2,2,0}=a_{2,0,2}=a_{2,3,0}=0$

(b) $a_{1,2,1}=-3 / 2 a_{2,3,0}, a_{2,1,2}=-9 / 2 a_{2,3,0}, a_{2,2,0}=a_{2,0,2}=0$

(c) $a_{1,2,1}=a_{2,2,0}=-1 / 2, a_{2,0,2}=1, a_{2,1,2}=-1, a_{2,3,0}=0$

(d) $a_{1,2,1}=-1 / 7, a_{2,2,0}=-1 / 2, a_{2,0,2}=1$,

$$
a_{2,3,0}=-1 / 14, a_{2,1,2}=-3 / 7
$$

2. $a_{1,0,3}=1$
(a) $a_{1,2,1}=-9 / 2, a_{2,1,2}=-3 / 2, a_{2,2,0}=a_{2,0,2}=a_{2,3,0}=0$
(b) $a_{2,3,0}=1, a_{1,2,1}=a_{2,1,2}=-3, a_{2,2,0}=a_{2,0,2}=0$
(c) $a_{2,0,2}=-3 / 2, a_{2,3,0}=0, a_{2,1,2}=a_{1,2,1}=a_{2,2,0}=0$
(d) $a_{2,0,2}=3 / 2, a_{2,3,0}=0, a_{2,1,2}=a_{1,2,1}=a_{2,2,0}=0$
(e) $a_{1,2,1}=-1, a_{2,2,0}=a_{2,0,2}= \pm \frac{\sqrt{2}}{2}, a_{2,1,2}=-2, a_{2,3,0}=0$,

3. $a_{1,0,3}=-1$

(a) $a_{2,3,0}=-1, a_{1,2,1}=a_{2,1,2}=3, a_{2,2,0}=a_{2,0,2}=0$

(b) $a_{1,2,1}=9 / 2, a_{2,1,2}=3 / 2, a_{2,3,0}=a_{2,2,0}=a_{2,0,2}=0$

In this proof, we do not present the algorithm generated polynomials which are too long.

Proof. We use the strategy given in lemma 3.2. We note also that we investigate only the real values of the parameters for which system (3.2) has an isochronous center at the origin.

1. Assume $a_{1,0,3}=0$ and then solve the isochronicity problem for system (3.2) under this assumption. We use the following change of coordinates $(x, y) \mapsto(-x, y)$ to obtain system (1.4) studied in [8]. The investigation following the two cases :

(a) $a_{2,0,2}=0$

(b) $a_{2,0,2}=1$

covers, (with respect to a linear change of coordinates), all the values of the parameters for which the center at the origin of system (1.4), see Remark 3.3. In [8], the author used Calgorithm which characterizes isochronicity by establishing associated Urabe function.

2. Consider the case $a_{1,0,3}=1$. Computations of normal forms of the initial system in polar form give (2.5)

$$
\dot{r}=\sum_{j=1}^{N} \alpha_{2 j+1} r^{2 j+1}+O\left(r^{2 N+3}\right), \dot{\theta}=1+\sum_{j=1}^{N} \beta_{2 j+1} r^{2 j}+O\left(r^{2 N+2}\right)
$$


we obtain in the radial component $\alpha_{2 j+1}=0$ until order $N=6$. So that the first six necessary conditions to have a center are satisfied. Analyzing isochronicity involves the angular component. Using $F G b$ for computing the Gröbner basis of the obtained first six quantities in the angular component, we obtain a Gröbner basis of 27 polynomials denoted $G_{1}$ such that it's first element is

$$
-a_{2,3,0} a_{2,2,0}\left(-a_{2,1,2}^{2}+9 a_{2,3,0}\right) .
$$

Then we analyze the isochronicity problem in the following three cases, which are given by the vanishing of one factor of the above expression.

(a) $a_{2,3,0}=0$, we substitute this condition into $G_{1}$ we compute again the associated Gröbner basis, we obtain a basis of 14 polynomials, when we solve it we obtain the following four real solutions to the problem :

(i) $a_{1,0,3}=1, a_{2,1,2}=-3 / 2, a_{1,2,1}=-9 / 2, a_{2,3,0}=a_{2,2,0}=a_{2,0,2}=0$

(ii) $a_{1,0,3}=1, a_{2,0,2}=3 / 2, a_{2,3,0}=a_{1,2,1}=a_{2,2,0}=a_{2,1,2}=0$

(iii) $a_{1,0,3}=1, a_{2,0,2}=-3 / 2, a_{2,3,0}=a_{1,2,1}=a_{2,2,0}=a_{2,1,2}=0$

(iv) $a_{1,0,3}=1, a_{1,2,1}=-1, a_{2,2,0}=a_{2,0,2}= \pm \frac{\sqrt{2}}{2}, a_{2,1,2}=-2$

(b) For $a_{2,2,0}=0$, substituting this assumption in $G_{1}$, and computing it's associated Gröbner basis which contains 7 polynomials, after solving it we obtain the solutions :

(i) $a_{1,0,3}=1, a_{2,0,2}=3 / 2, a_{2,2,0}=a_{1,2,1}=a_{2,3,0}=a_{2,1,2}=0$

(ii) $a_{1,0,3}=1, a_{2,0,2}=-3 / 2, a_{2,2,0}=a_{1,2,1}=a_{2,3,0}=a_{2,1,2}=0$

(iii) $a_{1,0,3}=1, a_{2,1,2}=-3 / 2, a_{1,2,1}=-9 / 2, a_{2,2,0}=a_{2,3,0}=a_{2,0,2}=0$

(iv) $a_{1,0,3}=a_{2,3,0}=1, a_{2,1,2}=a_{1,2,1}=-3, a_{2,2,0}=a_{2,0,2}=0$

(c) Similarly for $a_{2,3,0}=(1 / 9) a_{2,1,2}^{2}$, we obtain the solutions

(i) $a_{1,0,3}=1, a_{2,0,2}=-3 / 2, a_{2,2,0}=a_{1,2,1}=a_{2,3,0}=a_{2,1,2}=0$

(ii) $a_{1,0,3}=1, a_{2,0,2}=3 / 2, a_{2,2,0}=a_{1,2,1}=a_{2,3,0}=a_{2,1,2}=0$

(iii) $a_{1,0,3}=a_{2,3,0}=1, a_{2,1,2}=a_{1,2,1}=-3, a_{2,2,0}=a_{2,0,2}=0$

It's easy to see that several solutions are repeated above. For example cases (2(a)ii $\equiv$ 2(b)i) and (2(a)iii $\equiv 2$ (b)ii). We claim that there are only 5 solutions to the problem when $a_{1,0,3}=1$ which are given in the theorem.

Analysis of the theorem cases with $a_{1,0,3}=1$

- $2 \mathrm{a}$ - In this case system (3.2) reduces to

$$
\left.\begin{array}{l}
\dot{x}=y-9 / 2 y x^{2}+y^{3} \\
\dot{y}=-x-3 / 2 x y^{2}
\end{array}\right\}
$$

which is a cubic homogeneous perturbation of linear center with isochronous center at the origin. Indeed, we use the change of coordinates $(x, y) \mapsto\left(\frac{y}{\sqrt{2}}, \frac{x}{\sqrt{2}}\right)$ we obtain system $\tilde{S}^{*}{ }_{3}$ given in $[7,19]$. A first integral, a linearizing change of coordinate and a transversal commuting system are established for homogeneous perturbations (see[17, 7]).

- 2b-System (3.2) reduces to

$$
\left.\begin{array}{l}
\dot{x}=y-3 x^{2} y+y^{3} \\
\dot{y}=-x+x^{3}-3 x y^{2}
\end{array}\right\}
$$

which is an homogeneous perturbation of linear center, by the change of coordinates $(x, y) \mapsto(y, x)$ reduces to system $S^{*}{ }_{1}$ of $[19,17,7]$.

- $2 \mathrm{c}$ - and $2 \mathrm{~d}$ - For these two cases system (3.2) reduces to

$$
\left.\begin{array}{l}
\dot{x}=y+y^{3} \\
\dot{y}=-x \pm 3 / 2 y^{2}
\end{array}\right\}
$$


We see that by the change of coordinate $(x, y) \mapsto(y, x)$, we have a Liénard systems $x^{\prime \prime}+f(x) x^{\prime}+g(x)=0$ satisfying

$$
g(x)=g^{\prime}(0) x+\frac{1}{x^{3}}\left(\int_{0}^{x} s f(s) d s\right)^{2}
$$

then the origin is an isochronous center. See $[9,11,1]$ for more details about characterization of isochronicity for Liénard equation.

- 2e- In this case system (3.2) is a time- reversible system with an isochronous center at $O$. Indeed, in polar coordinates it reduces to

$$
\left.\begin{array}{l}
\dot{r}=-1 / 2 \sin (\theta) r^{2} \sqrt{2}+1 / 2 r^{3} \sin (2 \theta) \\
\dot{\theta}=1+(-1 / 2 \cos (2 \theta)+1 / 2) r^{2}-1 / 2 r \cos (\theta) \sqrt{2}
\end{array}\right\}
$$

which belongs to the family ii) (with $R_{1}=r_{1}=-\frac{\sqrt{2}}{2}$ if $a_{2,2,0}=a_{2,0,2}=\frac{\sqrt{2}}{2}$ ) and ( $R_{1}=r_{1}=\frac{\sqrt{2}}{2}$ if $a_{2,2,0}=a_{2,0,2}=-\frac{\sqrt{2}}{2}$ ) of the theorem 8.11 in Garcia thesis, see also system $C R_{4}$ of [7].

3. $a_{1,0,3}=-1$. When executing the normal form Maple code, there is no change from the case $a_{1,0,3}=1$ : the coefficients of radial component of system (2.5) are such that $\alpha_{2 j+1}=0$ until order $N=6$. The first six necessary conditions to have a center are satisfied. On a similar way as the case $a_{1,0,3}=1$, for analyzing isochronicity we are concerned by the angular component. We compute the Gröbner basis ,denoted $G_{-1}$, of the obtained first six quantities in the angular component. We obtain an ideal of 27 polynomials such that its first element is

$$
a_{2,2,0} a_{2,3,0}\left(a_{2,1,2}^{2}+9 a_{2,3,0}\right) \text {. }
$$

Then we analyze the isochronicity problem in the following three cases, which are the vanishing of each of the factors of the above expression.

(a) For $a_{2,3,0}=0$, we substitute this assumption in $G_{-1}$. We compute again the Gröbner basis associated to this case. We obtain a basis of 14 polynomials. when we solve it we obtain the unique real solution to the problem :

(i) $a_{1,0,3}=-1, a_{1,2,1}=9 / 2, a_{2,1,2}=3 / 2, a_{2,3,0}=a_{2,2,0}=a_{2,0,2}=0$

(b) For $a_{2,2,0}=0$ we do the same as for the last case and obtain the two real solutions

(i) $a_{1,0,3}=a_{2,3,0}=-1, a_{1,2,1}=a_{2,1,2}=3, a_{2,2,0}=a_{2,0,2}=0$

(ii) $a_{1,0,3}=-1, a_{1,2,1}=9 / 2, a_{2,1,2}=3 / 2, a_{2,3,0}=a_{2,2,0}=a_{2,0,2}=0$

(c) $a_{2,3,0}=-(1 / 9) a_{2,1,2}^{2}$ in the same way as for the first and second cases we obtain

(i) $a_{1,0,3}=a_{2,3,0}=-1, a_{1,2,1}=a_{2,1,2}=3, a_{2,2,0}=a_{2,0,2}=0$

We conclude that in the case $a_{1,0,3}=-1$, we have only two solutions to the isochronicity problem.

Analysis of the theorem cases with $a_{1,0,3}=-1$

These two solutions are cubic homogeneous perturbations of the linear center, which can be found in [7].

- 3a- in this case, system (3.2) can be written

$$
\left.\begin{array}{l}
\dot{x}=y+3 x^{2} y-y^{3} \\
\dot{y}=-x-x^{3}+3 x y^{2}
\end{array}\right\}
$$

by the change of coordinates $(x, y) \mapsto(-x,-y)$, it reduces to system $S^{*}{ }_{1}$ of $[19,17,7]$, which have an isochronous center at the origin.

- 3b- system (3.2) can be written

$$
\left.\begin{array}{l}
\dot{x}=y+9 / 2 y x^{2}-y^{3} \\
\dot{y}=-x+3 / 2 x y^{2}
\end{array}\right\}
$$


we use the change of coordinates $(x, y) \mapsto\left(\frac{y}{\sqrt{2}}, \frac{x}{\sqrt{2}}\right)$ we obtain system $S^{*}{ }_{3}$ with isochronous center at the origin $O$ given in $[7,19]$.

That ends the proof.

\subsection{Perturbation $a_{1,3,0}$}

Consider the system

$$
\left.\begin{array}{l}
\dot{x}=y+a_{1,2,1} x^{2} y+a_{1,3,0} x^{3} \\
\dot{y}=-x+a_{2,2,0} x^{2}+a_{2,0,2} y^{2}+a_{2,3,0} x^{3}+a_{2,1,2} x y^{2}
\end{array}\right\}
$$

Theorem 3.5. System (3.9) with $a_{1,3,0} \neq 0$ has no center at the origin. Moreover, system (3.9) has an isochronous center at the origin $O$ if and only if it reduces to system (1.4) and its parameters satisfy one of isochronicity cases from those of Theorem (1.1).

Proof. Analogously to lemma 3.2 we have to analyse $a_{1,3,0}= \pm 1$.

Executing the Maple code, which gives the normal form of (3.9) in it's polar form (2.5)

$$
\dot{r}=\sum_{j=1}^{N} \alpha_{2 j+1} r^{2 j+1}+O\left(r^{2 N+3}\right), \dot{\theta}=1+\sum_{j=1}^{N} \beta_{2 j+1} r^{2 j}+O\left(r^{2 N+2}\right)
$$

we obtain in the radial component such that $\alpha_{3}= \pm \frac{3}{8}$, then in this case, the singular point can not be a center.

\subsection{Perturbation $a_{2,0,3}$}

This perturbation represents system (1.4) perturbed by the additional monomial with the parameter $a_{2,0,3}$

$$
\left.\begin{array}{l}
\dot{x}=y+a_{1,2,1} x^{2} y \\
\dot{y}=-x+a_{2,2,0} x^{2}+a_{2,0,2} y^{2}+a_{2,3,0} x^{3}+a_{2,1,2} x y^{2}+a_{2,0,3} y^{3}
\end{array}\right\}
$$

Theorem 3.6. System (3.10) with $a_{2,0,3} \neq 0$ has no center at the origin. Moreover, system (3.10) has an isochronous center at $O$ if and only if it reduces to system (1.4) and its parameters satisfy one case of isochronicity conditions given in Theorem (1.1).

Proof. Analogously to lemma 3.2.

Consider $a_{2,0,3}= \pm 1$.

Executing the Maple code, which gives the normal form of (3.10) in it's polar form (2.5)

$$
\dot{r}=\sum_{j=1}^{N} \alpha_{2 j+1} r^{2 j+1}+O\left(r^{2 N+3}\right), \dot{\theta}=1+\sum_{j=1}^{N} \beta_{2 j+1} r^{2 j}+O\left(r^{2 N+2}\right)
$$

we obtain in the radial component such that $\alpha_{3}= \pm \frac{3}{8}$, then in this case, the singular point cant be a center.

\subsection{Perturbation $a_{1,1,2}$}

This case represents system (1.4) perturbed by the monomial with the parameter $a_{1,1,2}$

$$
\left.\begin{array}{l}
\dot{x}=y+a_{1,2,1} x^{2} y+a_{1,1,2} x y^{2} \\
\dot{y}=-x+a_{2,2,0} x^{2}+a_{2,0,2} y^{2}+a_{2,3,0} x^{3}+a_{2,1,2} x y^{2}
\end{array}\right\}
$$

Theorem 3.7. System (3.11) with $a_{1,1,2} \neq 0$ has no center at the origin. Moreover, system (3.11) has an isochronous center at $O$ if and only if it reduces to one case of isochronicity from those of system (1.4).

Proof. when $a_{1,1,2} \neq 0$ we obtain in the radial component $\alpha_{3}= \pm \frac{1}{8}$, then in this case, the singular point at the origin cant be a center. 


\subsection{Perturbation $a_{2,2,1}$}

This case represents system (1.4) perturbed by the monomial with the parameter $a_{2,2,1}$

$$
\left.\begin{array}{l}
\dot{x}=y+a_{1,2,1} x^{2} y \\
\dot{y}=-x+a_{2,2,0} x^{2}+a_{2,0,2} y^{2}+a_{2,3,0} x^{3}+a_{2,1,2} x y^{2}+\underline{a_{2,2,1} x^{2} y}
\end{array}\right\}
$$

Theorem 3.8. System (3.12) with $a_{2,2,1} \neq 0$ has no center at the origin. Moreover, system (3.12) has an isochronous center at $O$ if and only if it reduces to one case of isochronicity from those of system (1.4).

Proof. By the same reason from the one of the last case, we substitute $a_{2,2,1}= \pm 1$ in the system, then we obtain in the radial component $\alpha_{3}= \pm \frac{1}{8}$, in this case, the singular point at the origin can not be a center.

\subsection{Perturbation $a_{1,0,2}$}

This perturbation represents system (1.4) with an additional monomial $a_{1,0,2} y^{2}$ in the first equation

$$
\left.\begin{array}{l}
\dot{x}=y+a_{1,2,1} x^{2} y+\underline{a_{1,0,2} y^{2}} \\
\dot{y}=-x+a_{2,2,0} x^{2}+a_{2,0,2} y^{2}+a_{2,3,0} x^{3}+a_{2,1,2} x y^{2}
\end{array}\right\}
$$

Theorem 3.9. System (3.13) with $a_{1,0,2} \neq 0$ has no isochronous center at the origin. Moreover, system (3.13) has an isochronous center at $O$ if and only if $a_{1,0,2}=0$ and it reduces (modulo a linear change of coordinates) to system (1.4) such as its parameters satisfy one of the four cases given in Theorem 1.1.

Proof. Since we have a quadratic perturbation of system (1.4), thanks to Remark 1 we study the cases $a_{1,0,2} \in\{0,1\}$. If $a_{1,0,2}=0$, system (3.13) admit an isochronous center at the origin if and only if it reduces to (1.4) and its parameters satisfy one the isochronicity conditions given by Theorem 1.1. We assume $a_{1,0,2}=1$, and we compute first the radial component of the normal form in polar coordinates. The first radial component $\alpha_{3}=-a_{2,0,2} / 4$, then we substitute the assumption $a_{2,0,2}=0$ in the remaining five $\alpha_{2 j+1}$ and we compute the associated Gröbner base, we find $\alpha_{5}=$ $a_{2,2,0}\left(a_{2,1,2}+a_{1,2,1}\right)$. We continue the analysis in the three cases :

1. $a_{2,2,0}=0$

2. $\left(a_{2,1,2}+a_{1,2,1}\right)=0$

Unfortunately, we computed in each case the Gröbner base and there are no common roots between the multivariate polynomials $\beta_{2 j+1}$ of the angular component.

\subsection{Perturbation $a_{1,2,0}$}

Consider the system

$$
\left.\begin{array}{l}
\dot{x}=y+a_{1,2,1} x^{2} y+\underline{a_{1,2,0} x^{2}} \\
\dot{y}=-x+a_{2,2,0} x^{2}+a_{2,0,2} y^{2}+a_{2,3,0} x^{3}+a_{2,1,2} x y^{2}
\end{array}\right\}
$$

Theorem 3.10. System (3.14) with $a_{1,2,0} \neq 0$ has an isochronous center at $O$ if and only if, modulo a linear change of coordinates, its parameters satisfy

$$
a_{2,3,0}=-4 / 9, a_{2,1,2}=0, a_{1,2,1}=0, a_{2,2,0}=0, a_{2,0,2}=0, a_{1,2,0}=1
$$

Proof. Consider the case $a_{1,2,0}=1$. We compute first, under this assumption, the radial component of the normal form of system (3.14). We obtain $\alpha_{3}=a_{2,2,0} / 4$. Then the first necessary condition to have a center at the origin is the vanishing of $a_{2,2,0}$. We substitute this additional assumption in the remaining coefficients of the radial component $\left(\alpha_{5} \ldots \alpha_{13}\right)$. A common factor appears which is $a_{2,0,2}$. 
Hence we obtain two cases to analyse center conditions $a_{2,0,2}=0$ and $a_{2,0,2} \neq 0$.

For the case $a_{2,0,2} \neq 0$, we divide all the expressions of the coefficients of the radial component by $a_{2,0,2}$. We compute the associated Gröbner basis which is generated by 8 polynomials and gives 3 cases for each one the first six necessary conditions for the center are satisfied. Namely the following

$$
\begin{gathered}
\left\{a_{2,1,2}=0, a_{1,2,1}=0, a_{2,2,0}=0, a_{2,0,2}=0, a_{1,2,0}=1, a_{2,3,0}=-3 / 4\right\} \\
\left\{a_{2,2,0}=0, a_{1,2,0}=1, a_{2,3,0}=0, a_{2,0,2}=-1, a_{2,1,2}=-a_{1,2,1}\right\} \\
\left\{a_{2,2,0}=0, a_{1,2,0}=1, a_{2,3,0}=0, a_{2,1,2}=-a_{1,2,1}, a_{2,0,2}=1\right\}
\end{gathered}
$$

The first solution is rejected, because we have assumed that $a_{2,0,2} \neq 0$.

For the second and the third solution of center condition investigation, we substitute each of those into the angular component coefficients expressions. We compute the Gröbner bases of the obtained multivariate polynomial systems. Unfortunately in the two cases it gives Gröbner basis $\equiv[1]$ which means that there are not common roots.

We return to the remaining case $a_{1,2,0}=1, a_{2,2,0}=a_{2,0,2}=0$ which ensures the first six necessary conditions of the singular point at the origin to be a center. Substituting this assumption in the angular component coefficients and computing its associated Gröbner basis which is generated by three polynomials. This gives the unique solution to the problem of isochronicity

$$
a_{2,3,0}=-4 / 9, a_{1,2,0}=1, a_{2,1,2}=a_{1,2,1}=a_{2,2,0}=a_{2,0,2}=0
$$

Then system (3.14) reduces to

$$
\left.\begin{array}{l}
\dot{x}=y+x^{2} \\
\dot{y}=-x-4 / 9 x^{3}
\end{array}\right\}
$$

Which is a Liénard isochronous system with $f(x)=-2 x, g(x)=x+4 / 9 x^{3}$ satisfying

$$
g(x)=g^{\prime}(0) x+\frac{1}{x^{3}}\left(\int_{0}^{x} s f(s) d s\right)^{2}
$$

3.9. Perturbation $a_{2,1,1}$

$$
\left.\begin{array}{l}
\dot{x}=y+a_{1,2,1} x^{2} y \\
\dot{y}=-x+a_{2,2,0} x^{2}+a_{2,0,2} y^{2}+\underline{a_{2,1,1} x y}+a_{2,3,0} x^{3}+a_{2,1,2} x y^{2}
\end{array}\right\}
$$

Theorem 3.11. System (3.15) with $a_{2,1,1} \neq 0$ has an isochronous center at $O$ if and only if its parameters satisfy one of the following two cases

1. $a_{2,1,1}=1, a_{2,3,0}=-1 / 9, a_{2,1,2}=a_{1,2,1}=a_{2,2,0}=a_{2,0,2}=0$

2. $a_{2,1,1}=1, a_{2,1,2}=-2 / 9, a_{1,2,1}=1 / 9, a_{2,2,0}=a_{2,0,2}=a_{2,3,0}=0$

Proof. Consider the $a_{2,1,1}=1$

We compute first, under this assumption, the radial component of the normal form of system (3.15). We obtain $\alpha_{3}=\left(a_{2,0,2}+a_{2,2,0}\right) / 8$, then the first necessary condition to have a center at the origin is the vanishing of $\alpha_{3}$.

We substitute this additional assumption $a_{2,0,2}=-a_{2,2,0}$ in the following coefficient of the radial component $\alpha_{5}=-1 / 48 a_{2,0,2}\left(a_{1,2,1}-a_{2,1,2}+a_{2,3,0}\right)$, then it appears two cases to analyze: $\left\{a_{2,0,2}=a_{2,2,0}=0\right\}$ and $\left\{a_{2,0,2}=-a_{2,2,0}, a_{1,2,1}-a_{2,1,2}+a_{2,3,0}=0\right\}$

1. $a_{2,0,2}=a_{2,2,0}=0$ We substitute this additional assumption in the remaining coefficients of the radial component $\alpha_{7}, \ldots, \alpha_{13}$ which gives $\alpha_{3}=\alpha_{5} \ldots=\alpha_{13}=0$. Hence, the first six center necessary conditions are satisfied. 
Substituting the assumptions into the angular component coefficients expressions, computing associated Gröbner basis gives the two real solutions. The first one is

$$
\left\{a_{2,1,1}=1, a_{2,3,0}=-1 / 9, a_{2,1,2}=a_{2,2,0}=a_{2,0,2}=a_{1,2,1}=0\right\}
$$

under which system (3.15) reduces to

$$
\left.\begin{array}{l}
\dot{x}=y \\
\dot{y}=-x+x y-1 / 9 x^{3}
\end{array}\right\}
$$

we have an isochronous Liénard systems with

$$
f(x)=-x \text { and } g(x)=x+1 / 9 x^{3}
$$

which satisfy

$$
g(x)=g^{\prime}(0) x+\frac{1}{x^{3}}\left(\int_{0}^{x} s f(s) d s\right)^{2}
$$

The second one is

$$
\left\{a_{2,2,0}=0, a_{2,0,2}=0, a_{2,1,1}=1, a_{2,3,0}=0, a_{2,1,2}=-2 / 9, a_{1,2,1}=1 / 9\right\}
$$

under which system (3.15) reduces to

$$
\left.\begin{array}{l}
\dot{x}=y+1 / 9 x^{2} y \\
\dot{y}=-x+x y-2 / 9 x y^{2}
\end{array}\right\}
$$

By the change of coordinates $(x, y) \mapsto(y, x)$ we obtain

$$
\left.\begin{array}{l}
\dot{x}=-y+x y-2 / 9 x^{2} y \\
\dot{y}=x+1 / 9 x y^{2}
\end{array}\right\}
$$

Which belongs to the Liénard Type equation (1.2) with $f(x)=-3(2 x-3)^{-1}$ and $g(x)=$ $1 / 9 x(2 x-3)(x-3)$. The isochronicity of this last system is proved since it belongs to the case 9 of the Theorem 3 of Chouikha et.al in [10].

2. $a_{2,0,2}=-a_{2,2,0}$ and $a_{1,2,1}-a_{2,1,2}+a_{2,3,0}=0$

Unfortunately in this case, after computing Gröbner basis, no real solutions are found.

Theorem 3.12. System (1.4) has an amplitude independent frequency synchronizer at $O$ if and only if its parameters satisfy one of the cases of Theorem 3-Theorem 11.

To summarize, for the eight monomial perturbations studied in this paper we have identified all possible amplitude independent frequency synchronizer. Moreover, we claim that all isochronous cases are known in a fragmented literature but our study insures that for the studied family there are no other (necessary conditions). Each of them was found in a classification of a specific family of planar differential systems which are different from the context of the ones studied in this paper (synchronization). For isochronous centers of Liénard systems the reader can see for instance [11,7]. The papers $[8,10]$ are concerned with the planar differential Liénard Type equations. For cubic time reversible systems see $[7,15,12]$ and for cubic homogeneous perturbations of linear center see $[7,15,17]$. 


\section{References}

[1] A. Algaba, E. Freire, E. Gamero, Isochronicity via normal form, Qual. Theory Of Dyn. Systems. 1, p. 133-156, (2000).

[2] M. Bardet, I. Boussaada, A.R. Chouikha, J-M. Strelcyn, Isochronicity conditions for some planar polynomial systems II, Bull. Sci. Math, 135, Issue 2, (2011), Pages 230-249.

[3] M. Bardet, I. Boussaada, Compexity reduction of C-algorithm App. Math. and Comp., In Press, Available online, (2011).

[4] T. Becker, V. Weispfenning, Gröbner bases, Springer-Verlag, (1993).

[5] I. I. Blekhman, Synchronisation in Science and Technology, ASME Press translations, New York, 1988

[6] I. Boussaada, A.R. Chouikha, J-M. Strelcyn, Isochronicity conditions for some planar polynomial systems, Bull. Sci. Math, 135, Issue 1, (2011), Pages 89-112.

[7] J. Chavarriga, M. Sabatini, A survey of isochronous centers, Qual. Theory of Dyn. Systems vol 1, (1999), $1-70$.

[8] A. R. Chouikha, Isochronous centers of Lienard type equations and applications, J. Math. Anal. Appl. 331 (2007), 358-376 .

[9] A. R. Chouikha, Monotonicity of the period function for some planar differential systems. Part II: Liénard and related systems, Applicationes Mathematicae, 32 no. 4 (2005), 405-424.

[10] A. R. Chouikha,V. G. Romanovski, X. Chen Isochronicity of analytic systems via Urabe's criterion, $J$. Phys. A, 40 (2007) 10, 2313-2327.

[11] C. Christopher and J. Devlin, On the classification of Lienard systems with amplitude-independent periods, J. Diff. Eq. (2004), 1-17.

[12] X. Chen, V.G. Romanovski, Linearizability conditions of time-reversible cubic systems, J. Math. Anal. Appl., 362, (2010), N:2,p. 438-449,

[13] J. C. Faugère, FGb Salsa Software, http://fgbrs.lip6.fr

[14] J-P. Françoise, Isochronous Systems and Perturbation Theory, Journal of Nonlinear Mathematical Physics V: 12, (2005), 315326.

[15] I. Garcia, Contribution to the qualitative study of planar vector fields, PhD Thesis, Dept. de Matematica. University of Lleida (2000).

[16] J. Guckenheimer, P. Holmes, Nonlinear oscillations, dynamical systems, and bifurcation of vector fields Springer, 2002. xvi+459 pp.

[17] P. Mardesić, C. Rousseau, B. Toni, Linearization of isochronous Centers, J. Diff. Eq. 121, p.67-108 (1995).

[18] A. Pikovsky, M. Rosenblum and J. Kurths, Synchronization: A Universal Concept in Nonlinear Sciences. Cambridge Nonlinear Science Series, 2003.

[19] I. I. Pleshkan, A new method of investigating the isochronicity of a system of two differential equations, Diff. Eq.5, p. 796-802 (1969).

[20] M. Sabatini, On the period function of Lienard systems, J. Diff. Eq. 152, p. 467-487, 152 (1999).

[21] M. Sabatini, On the period function of $x^{\prime \prime}+f(x) x^{2}+g(x)=0$, J. Diff. Eq. 196, no. 1, p. 151-168 (2004).

[22] P. Yu, Q. Bi Symbolic computation of normal forms for semi-simple case, J. Comput. Appl. Math. 102, (1999) p. 195-220.

[23] P. Yu, Computation of normal forms via a perturbation technique, J. Sound Vibration p.19-38, 211(1998).

[24] P. Yu, A. Y. T. Leung Normal forms of vector field with perturbation parameters and their application, Chaos Solitons Fractals p.564-579, 34 (2007). 


\section{Acknowledgment}

Financial support by the Institut Polytechnique des Sciences Avancées is gratefully acknowledged. I warmly thank Silviu-Iulian Niculescu and Hugues Mounier who indroduced me to Engineering Sciences during my Post-doctoral stay at L2S, CNRS-Supélec-Université Paris Sud. I am grateful to A.Raouf Chouikha (Université Paris 13, France) and Jean-Marie Strelcyn (Université de Rouen, France) and Andrzej Maciejewski (University of Zielona Gòra, Poland) for many important hints and fruitful discussions. I am also indebted to Antonio Loria for constructive discussions about the synchronization problem during an advanced course in this topic organized by the EECI-HYCON2 Graduate School on Control held in january 2011 at L2S. Last but not least, I thank the anonymous referees for their suggestions.

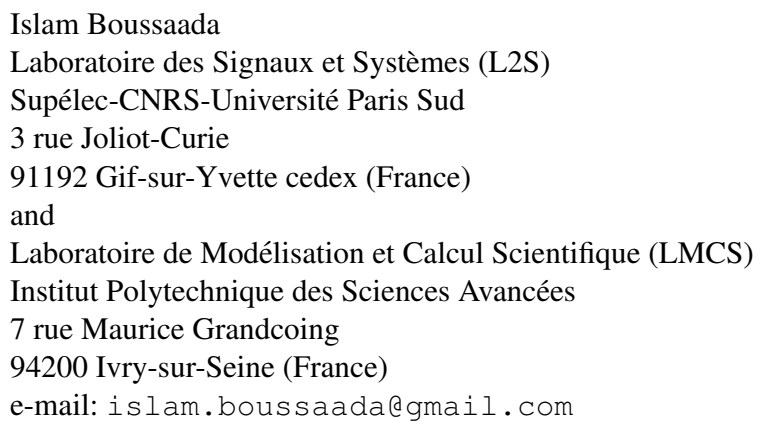

